\title{
A Feasibility Test on the DGPS by Correction Projection Using MSAS Correction
}

\author{
Dong Hwan Yoon', Byungwoon Park ${ }^{1 \dagger}$, Ho Yun², Changdon Kee ${ }^{2}$ \\ ${ }^{1}$ Department of Aerospace Engineering, Sejong University, Seoul 143-747, Korea \\ ${ }^{2}$ School of Mechanical and Aerospace Engineering and SNU-IAMD, Seoul National University, Seoul 151-744, Korea
}

\begin{abstract}
Differential Global Positioning System-Correction Projection (DGPS-CP) algorithm, which has been suggested as a method of correcting pre-calculated position error by projecting range-domain correction to positional domain, is a method to improve the accuracy performance of a low price GPS receiver to 1 to $3 \mathrm{~m}$, which is equivalent to that of DGPS, just by using a software program without changing the hardware. However, when DGPS-CP algorithm is actually realized, the error is not completely eliminated in a case where a reference station does not provide correction of some satellites among the visible satellites used in user positioning. In this study, the problem of decreased performance due to the difference in visible satellites between a user and a reference station was solved by applying the Multifunctional Transport Satellites (MTSAT) based Augmentation System (MASA) correction to DGPS-CP, instead of local DGPS correction, by using the Satellite Based Augmentation System (SBAS) operated in Japan. The experimental results showed that the accuracy was improved by $25 \mathrm{~cm}$ in the horizontal root mean square (RMS) and by $20 \mathrm{~cm}$ in the vertical RMS in comparison to that of the conventional DGPS-CP.
\end{abstract}

Keywords: DGPS, DGPS-CP, NMEA format, MSAS

\section{INTRODUCTION}

Differential global positioning system (DGPS) is a method of improving positioning accuracy to a level of 1 to $3 \mathrm{~m}$ by applying to a user's receiver the correction generated from a reference station of which accurate position is measured, on the basis of the fact that common error of the signals received from the GPS has a great temporal and spatial correlation (Kaplan 2006).

Although a position-domain DGPS is very intuitive and easy to realize, it may have an error which is rather greater than that of a stand-alone system in a case where the visible satellites of a user are different from those of a reference station (Rizos 1999). Hence, range-domain correction, in which correction is selected according to the visible satellites of a user to remove the error, is generally used. The

Received Jan 22, 2014 Revised Feb 06, 2014 Accepted Feb 14, 2014

†Corresponding Author

E-mail: byungwoon@sejong.ac.kr

Tel: +82-2-3408-4385 Fax: +82-2-3408-3333
Satellite Based Augmentation System (SBAS) and the Radio Technical Commission for Maritime (RTCM) Services of the National DGPS (NDGPS), which are representative DGPS methods, all adopt the range-domain correction.

Applying the DGPS range-domain correction requires a DGPS function embedded in a receiver or a GPS receiver module enabling output of raw data. However, since low-price GPS modules provide as output only the basic elements for applications such as present time and position, it is unavoidable for a low-price GPS instrument to employ the position-domain DGPS method in which positiondomain error is directly removed. To appropriately apply the position-domain DGPS to a low-price instrument, recently suggested is DGPS-carrier phase (DGPS-CP) which calculates a position-domain correction vector by projecting the range-domain correction to the position-domain and enables a user to directly improve the accuracy by using the position-domain correction vector (Park et al. 2011).

The early DGPS-CP method required a separate communication to provide ephemeris, having the drawback that a communication module for the transmission 
Table 1. NMEA 0183 format (NMEA 2011).

\begin{tabular}{|c|c|c|}
\hline Type & Description & Example \\
\hline GPGGA & GPS fix data & $\begin{array}{l}\text { \$GPGGA, 123519, 4807.038,N, } \\
01131.000, \mathrm{E}, 1,08,0.9 \\
545.4, \mathrm{M}, 46.9, \mathrm{M},{ }^{*} 47\end{array}$ \\
\hline GPGLL & $\begin{array}{l}\text { Geographic position, } \\
\text { Latitude / Longitude }\end{array}$ & $\begin{array}{l}\text { \$GPGLL,4916.45,N,12311.12,W,225444,A, } \\
{ }^{* 1 D}\end{array}$ \\
\hline GPGSA & $\begin{array}{l}\text { GPS DOP and active } \\
\text { satellite }\end{array}$ & $\begin{array}{l}\text { \$GPGSA } \\
\text {,A,3,03,04,,09,12,,,24,,,,2.5,1.3,2.1*39 }\end{array}$ \\
\hline GPGSV & GPS Satellites in view & $\begin{array}{l}\text { \$GPG } \\
\text { SV,3,1,11,03,03,111,00,04,15,270,00,06,01,010, } \\
00,13,06,292,00 * 74\end{array}$ \\
\hline
\end{tabular}

of ephemeris should be installed in addition to the conventional DGPS infrastructure. To improve the drawback, it was shown that satellite constellation information included in GPS Satellite in View (GPGSV) information of the National Marine Electronics Association (NMEA) showed Table 1 (NMEA 2011) was used to constitute a line-of-sight vector on a local frame using the elevation angle and the azimuth angle information of a satellite and that an Earth Centered Earth Fixed (ECEF) coordinate-based line-of-sight matrix was constituted by a matrix rotation according to a user's latitude and longitude so that a low-price module could realize a performance level similar to that of the conventional DGPS without an additional data communication module (Yoon et al. 2013).

However, there still remains a limitation that the error may not be completely removed in a case where the visible satellites provided a local DGPS correction are different from the visible satellites of the user (Park et al. 2013). On the other hand, when an SBAS message is used, a Pseudo Range Correction (PRC) of all visible satellites may be generated with reference to a user position. In this article, therefore, a DGPS-CP method using the SBAS correction information was suggested. In addition, the effect of the DGPS-CP method was analyzed by applying the Japanese MSAS correction information which may be received in the region of South Korea.

\section{DGPS-CP IMPLEMENTATION USING MSAS CORRECTION}

\subsection{Multifunctional Transport Satellites (MTSAT) based Augmentation System (MASA)}

The SBAS, which is aimed at the improvement of aircraft navigation accuracy and integrity, includes the Wide Area Augmentation System (WAAS) of the U.S., the European Geostationary Navigation Overlay Service (EGNOS) of the Europe, the GPS Aided Geo-Augmented

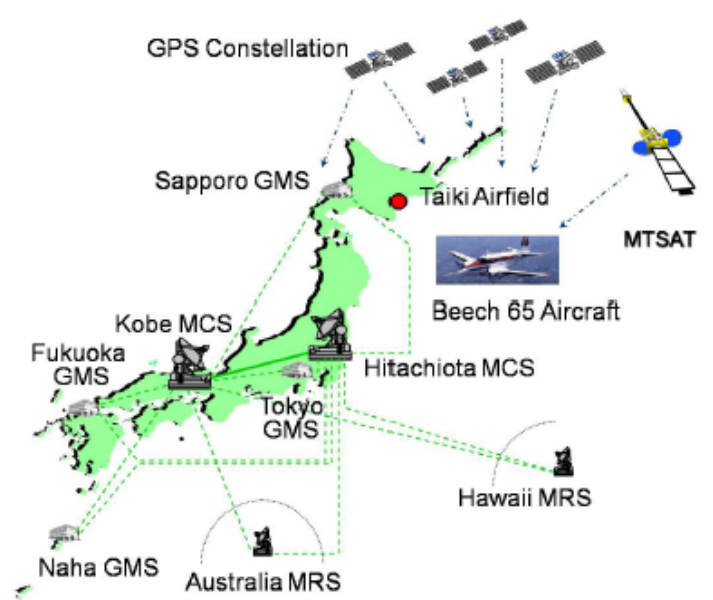

Fig. 1. MSAS system (Tsujii et al. 2008).

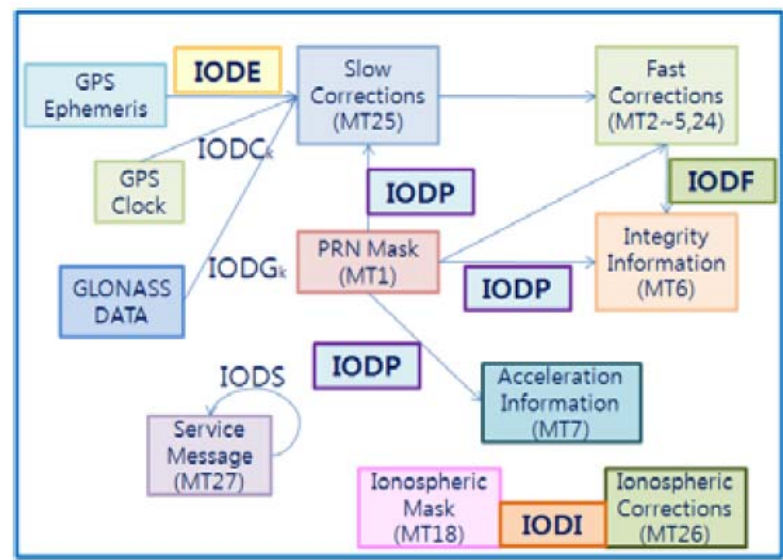

Fig. 2. SBAS message flow chart (RTCA 2001).

Navigation (GAGAN) of India, and the MTSAT Satellitebased Augmentation System (MSAS) of Japan, all of which are currently in operation. The SBAS is extensively used in all instruments employing GPS because the SBAS allows for receiving of correction by using the conventional GPS antenna. The MSAS was developed to provide correction to the aircrafts in the region of Japan using GPS. The MSAS basically provides PRC, ephemeris correction, and ionosphere correction through satellites (Shimamura 1999). Fig. 1 is a schematic diagram of the entire MSAS.

Fig. 2 shows the message type flow chart of the MSAS (RTCA 2001), and Table 2 shows the message time (Sakai et al. 2010).

MTs 2 to 5 transmit the PRC, Issue of Data PRN Mask (IODP), and Issue of Data Fast Correction (IODF) information of each GNSS. MT6 transmits the integrity information and the IODF information in the case where MTs 2 to 5 fail to receive the information in a predetermined time interval. 
Table 2. MSAS message type and schedule.

\begin{tabular}{ccccc}
\hline $\begin{array}{c}\text { Message } \\
\text { type }\end{array}$ & Component & $\begin{array}{c}\text { Timeout } \\
\text { interval for } \\
\text { NPA mode }\end{array}$ & $\begin{array}{c}\text { Timeout } \\
\text { interval for PA } \\
\text { mode }\end{array}$ & $\begin{array}{c}\text { Max } \\
\text { interval }\end{array}$ \\
\hline 2 to 5 & $\begin{array}{c}\text { Fast } \\
\text { Correction }\end{array}$ & 18 to 180s & 12 to 120s & 6 to 60s \\
\hline 24 & UDRE & 18 & 12 & 6 \\
\hline 6 & UDRE & 18 & 12 & 6 \\
\hline 9 & GEO Nav data & 360 & 240 & 120 \\
\hline $24 / 25$ & $\begin{array}{c}\text { Long-term } \\
\text { Correction }\end{array}$ & 360 & 240 & 120 \\
\hline 26 & $\begin{array}{c}\text { Ionospheric } \\
\text { Correction }\end{array}$ & 600 & 600 & 300 \\
\hline 28 & $\begin{array}{c}\text { Clock-ephemeris } \\
\text { Covariance }\end{array}$ & 360 & 240 & 120 \\
\hline
\end{tabular}

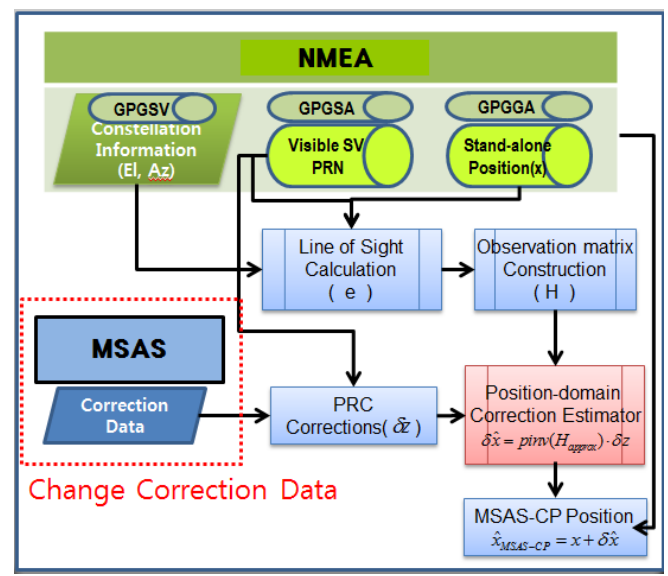

Fig. 3. MSAS-CP implementation method using satellite data in NMEA without ephemeris.

MTs 24 and 25 transmit the correction information with respect to satellite position and satellite clock error together with the IODP and Issue of Data (IOD). MT26 provides ionosphere correction and MT 28 provides the information related the PRC according to the satellite observation region and the increment of the User Differential Range Error (UDRE), which is the ephemeris correction estimation error so that the user may directly calculate the UDRE increment (Jeong 2009).

\subsection{DGPS-CP Implementation Using MSAS Correction}

Fig. 3 illustrates the method of applying the MSAS correction described in Section 2.1 to the DGPS-CP algorithm.

The DGPS-CP algorithm may be realized simply by replacing the conventional DGPS correction with the MSAS correction.

The PRC of each satellite may be calculated by using the SBAS correction provided by the MSAS as shown in Eq. (1):

$$
\delta \rho_{M S A S}^{i}=P R C^{i}=F C^{i}+I C^{i}+T C^{i}
$$

where in $F C^{i}, I C^{i}$, and $T C^{i}$ respectively denote the fast correction, ionosphere correction, and tropospheric correction of the ith satellite. Although the correction generated at a reference station is used by a user without modification in the general DGPS, a user who receives the SBAS correction may generate correction for all visible satellites at each position by combining the received information. Therefore, the problem of local DGPS in which correction of some satellites is lacked may be solved.

The generated correction is applied to the user measurement as shown in Eq. (2):

$$
\rho_{\text {corrected }}^{i}=\rho^{i}+\delta \rho_{M S A S}^{i}
$$

When the measurement in which error has been removed $\left(\rho_{\text {corrected }}^{i}\right)$ is applied to the observation equation, the equation is expanded as shown in Eq. (3) wherein $H$ denotes line-of-sight matrix, $B$ denotes the receiver clock error, and $e^{i}, \vec{R}^{i}, \rho^{i}$, and $\delta \vec{\rho}_{M S A S}^{i}$ respectively denote the line-of-sight vector, position vector, pseudo range, and the MSAS correction of the ith satellite:

$\left[\begin{array}{c}\stackrel{r}{M}_{M S A S} \\ B\end{array}\right]=\left(H^{T} H\right)^{-1} H\left[\begin{array}{c}\underset{r}{i} \\ e^{i} \mathscr{R}^{i}-\rho^{i} \\ \vdots\end{array}\right]+\left(H^{T} H\right)^{-1} H^{T}\left[\begin{array}{c}\vdots \\ -\delta \rho_{M S A S}^{i} \\ \vdots\end{array}\right]$

Since the left side of Eq. (3) is the same with the standalone position $(\vec{x})$, the MSAS correction is projected to the position-domain and the position-domain correction $\left(\delta \vec{x}_{M S A S}\right)$ is calculated through the correlation as shown in Eq. (4):

$$
\left[\begin{array}{c}
\delta \vec{x}_{M S A S} \\
\delta B
\end{array}\right]=\left(H^{T} H\right)^{-1} H^{T}\left[\begin{array}{c}
\vdots \\
-\delta \rho_{M S A S}^{i} \\
\vdots
\end{array}\right]=-\left(H^{T} H\right)^{-1} H^{T}\left(\delta \vec{\rho}_{M S A S}\right)
$$

Eq. (3) eventually turns to the position-domain correction shown in Eq. (4) which applies the position-domain correction $\left(\delta \vec{x}_{M S A S}\right)$ to the left side of Eq. (3), which is the stand-alone position $(\vec{x})$. Eq. (5) removes the error element from the position-domain and thereby enables DGPS in the position-domain.

$$
\vec{x}_{M S A S-C P}=\vec{x}+\delta \vec{x}_{M S A S}
$$

Therefore, the MSAS by correction projection expressed in Eq. (5) allows for obtaining the user position corrected by 


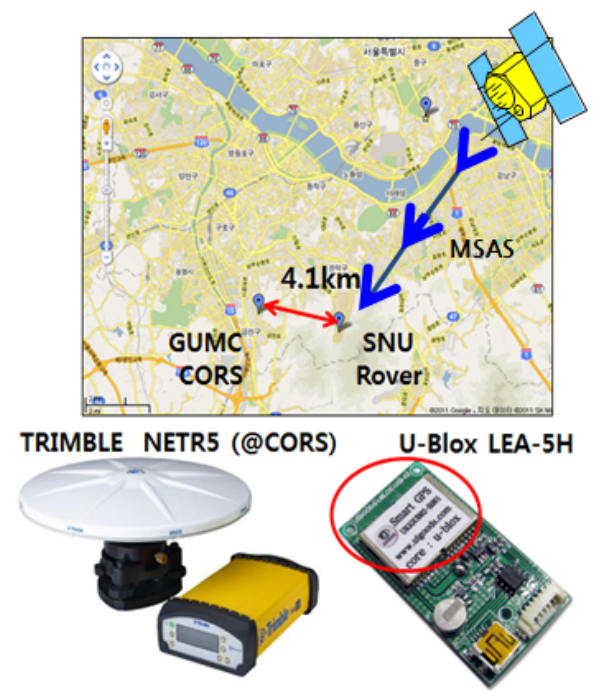

Fig. 4. MSAS-CP test construction.

the MSAS correction ( $\vec{x}_{\text {MSAS-CP }}$ ) by the same method with that of the DGPS-CP.

\section{PERFORMANCE OF MSAS-CP}

\subsection{Test construction of MSAS-CP}

To compare the results of applying the MSAS correction to the DGPS-CP with the result of the local DGPS, the U-blox LEA-5H receiver, which was used for observation on January 31, 2011, at Seoul National University, Seoul, Korea, and which stored the observation data, was employed as a user. The U-blox includes several positioning modes. Since the SBAS-off mode is similar to the stand-alone mode, the positioning mode was set to the SBAS-off mode in this study so that the logging data could be used as the position data without correction. As shown in Fig. 4, the data obtained at the Seoul Network Real Time Kinematic (RTK) GUMC (Gumcheon) Continuously Operating Reference Station, which was located about $4.1 \mathrm{~km}$ away from the user position, were used as the reference station data for the local DGPS. The PRC was generated in an interval of 30 seconds, which was the logging period of the reference station. The MSAS correction was generated by applying the MSAS messages stored on the observation date to all the visible satellites from the user position. The period of the MSAS correction generation was set to be 30 seconds, which was the same with the local DGPS condition. To differentiate the two different methods, the conventional local DGPS is referred to as DGPS-CP, while the method of applying the MSAS correction to the DGPS-CP is referred to as MSAS-CP.

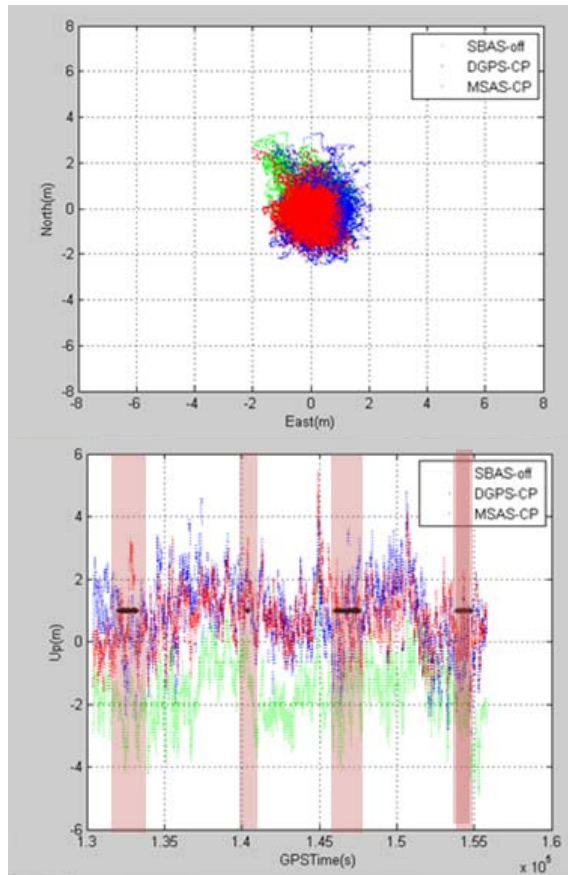

Fig. 5. DGPS-CP and MSAS-CP position result for 7 hours (Korean Standard Time 21:13:01 04:16:25) (Left: horizontal, Right: vertical).

Table 3. DGPS-CP and MSAS-CP result statistics.

\begin{tabular}{cccccccc}
\hline & \multicolumn{3}{c}{ Horizontal } & \multicolumn{3}{c}{ Vertical } \\
\cline { 2 - 8 } & $\begin{array}{c}\text { Stand-alone } \\
\text { (SBAS-off) }\end{array}$ & $\begin{array}{c}\text { DGPS- } \\
\text { CP }\end{array}$ & $\begin{array}{c}\text { MSAS- } \\
\text { CP }\end{array}$ & $\begin{array}{c}\text { Stand-alone } \\
\text { (SBAS-off) }\end{array}$ & $\begin{array}{c}\text { DGPS- } \\
\text { CP }\end{array}$ & $\begin{array}{c}\text { MSAS- } \\
\text { CP }\end{array}$ \\
\hline Mean (m) & 0.4576 & 0.3933 & 0.1440 & -1.6330 & 0.8624 & 0.7683 \\
\hline $\begin{array}{c}\text { Standard } \\
\text { Deviation } \\
(\mathrm{m})\end{array}$ & 0.8931 & 0.9771 & 0.7875 & 1.0225 & 1.1317 & 0.9582 \\
\hline RMS (m) & 1.0035 & 1.0533 & 0.8005 & 1.6921 & 1.1699 & 0.9797 \\
\hline
\end{tabular}

\subsection{Performance comparison between MSAS-CP and DGPS-CP}

The data stored on January 31, 2011 indicated that the time period when there was a great difference in the visible satellites between the user and the reference station due to the distance of $4 \mathrm{~km}$ between the two positions was from 21 o'clock to 4 o'clock in Korean Standard Time. Fig. 5 compares the results of applying the two MSAS-CP and DGPS-CP algorithms to the SBAS-off mode data of that time period.

Since the time period which is concern of this study is from night to dawn when the ionospheric error is not great, the effect of improving accuracy by applying the correction is not great. However, Table 3 shows that the mean horizontal error and the mean vertical error were decreased from $0.46 \mathrm{~m}$ and $-1.63 \mathrm{~m}$ to $0.14 \mathrm{~m}$ (MSAS-CP)/0.39 m 
(DGPS-CP) and $0.77 \mathrm{~m}$ (MSAS-CP)/ $0.86 \mathrm{~m}$ (DGPS-CP), respectively, indicating that both the MSAS-CP and the local DGPS algorithms may appropriately correct the positional error. While the local DGPS algorithm removed about $0.52 \mathrm{~m}(31 \%)$ of the vertical error RMS, the MSAS-CP correction algorithm removed about $0.71 \mathrm{~m}$ (43\%) of the vertical error RMS, indicating that the MSAS-CP algorithm effectively removed the positional error of the SBAS-off mode. This was because the local DGPS correction was not obtained with respect to some of the visible satellites used in the positioning in some time intervals. Fig. 5 shows the distribution of the vertical positional error by shading the time intervals.

In the first shaded interval, Satellites No. 12, 14, 18, 22, 25, 31 , and 32 were used in the positioning by the user receiver. However, the PRC generated at the GUMC reference station missed the Satellite No. 32 and the data from the Satellite No. 18 was also missed momentously In the third shaded interval, Satellites No. 3, 6, 13, 16, 19, 21, 23, 30, and 31were used but the correction of the Satellite No. 19 could not be applied. The analysis showed that the third shaded interval among the four shaded intervals was the most affected by the absence of the Satellite No. 19. Therefore, as shown in Fig. 6, the analysis of this study was focused on the time interval between GPS Time 146066 and 147599 (Korean Standard Time, 22:47:31 23:13:04) corresponding to the third shaded time interval.

According to Fig. 6, the mean vertical error included in the SBAS-off positioning was $-1.71 \mathrm{~m}$ but the bias of the SBAS-off was appropriately removed by correcting the error by projecting the MSAS and the local DGPS correction to the position-domain. The mean MSAS-CP vertical error was $0.85 \mathrm{~m}$ and the mean DGPS-CP error was $1.18 \mathrm{~m}$, indicating that there was not a significant difference between the two

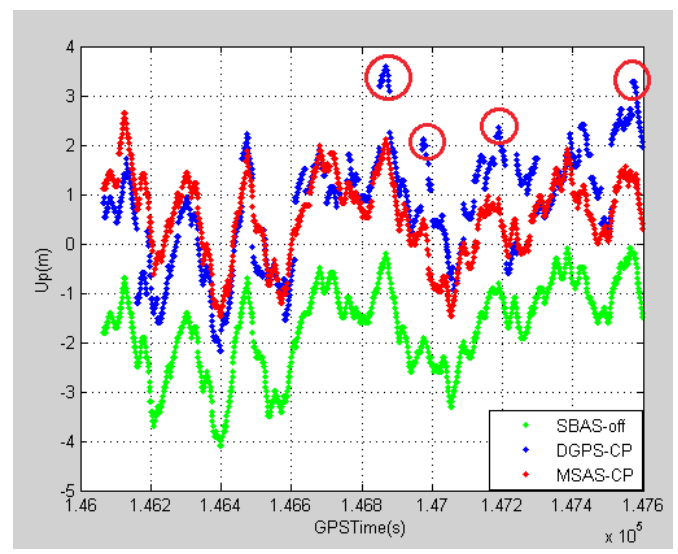

Fig. 6. DGPS-CP and MSAS-CP vertical error from 146066 to $147599 \mathrm{sec}$ (Korean Standard Time, 22:47:31 23:13:04). methods. However, as shown in Table 4, the error difference between the two methods might temporarily be more than $1.5 \mathrm{~m}$.

In addition, the MSAS-CP showed a continuous positional pattern as in the case of the stand-alone results. This result might be because the visible satellites used for positioning in the MSAS correction and in the stand-alone system were the same and thus the correction positiondomain projection was likewise varied continuously in the interval. On the other hand, the DGPS-CP results of by the local DGPS were discontinuous and included an error greater than that of the MSAS-CP. This was because the position correction was varied discontinuously due to the change of the visible satellites and the error included in the stand-alone position was not completely removed due to the discordance of the visible satellites between the reference station and the user. Table 5 shows the statistical data of the errors in the time interval where there is a discrepancy in the visible satellites between the local DGPS correction and the user. Table 5 shows the RMS values with respect to only the intervals where there is a discrepancy in the visible satellites between the local DGPS correction and the user. Table 5 also shows that the error removal performance of the MSAS-CP was $0.15 \mathrm{~m}$ in the horizontal direction and 0.04 $m$ in the vertical direction, indicating that the error removal performance of the MSAS-CP was better than that of the DGPS-CP.

\section{CONCLUSIONS}

In this study, the correction provided by the SBAS was applied to the DGPS-CP algorithm which is able to provide service of an accuracy level similar to that of the

Table 4. Vertical error differences between DGPS-CP and MSAS-CP due to the loss of local DGPS correction for a specific satellite.

\begin{tabular}{ccc}
\hline GPSTime (sec) / (KST) & DGPS-CP (m) & MSAS-CP (m) \\
\hline $146869(01: 47: 34)$ & 3.6031 & 2.1162 \\
$146974(01: 49: 19)$ & 2.1080 & 0.4981 \\
$147190(01: 52: 55)$ & 2.3532 & 0.8081 \\
$147575(01: 59: 20)$ & 3.2663 & 1.3540 \\
\hline
\end{tabular}

Table 5. DGPS-CP and MSAS-CP position accuracy during the period of the loss of local DGPS correction for a specific satellite.

\begin{tabular}{|c|c|c|c|c|c|c|}
\hline & \multicolumn{3}{|c|}{ Horizontal } & \multicolumn{3}{|c|}{ Vertical } \\
\hline & $\begin{array}{l}\text { Stand-alone } \\
\text { (SBAS-off) }\end{array}$ & $\begin{array}{c}\text { DGPS- } \\
\text { CP }\end{array}$ & $\begin{array}{c}\text { MSAS- } \\
\text { CP }\end{array}$ & $\begin{array}{c}\text { Stand-alone } \\
\text { (SBAS-off) }\end{array}$ & $\begin{array}{c}\text { DGPS- } \\
\text { CP }\end{array}$ & $\begin{array}{c}\text { MSAS- } \\
\text { CP }\end{array}$ \\
\hline RMS (m) & 1.0922 & 1.1917 & 1.0481 & 2.0312 & 0.9784 & 0.9395 \\
\hline
\end{tabular}


DGPS by using only the NMEA information and the result was compared with the result obtained by applying the conventional local DGPS correction. In this study, the MSAS correction was applied in replacement of the local DGPS correction to a U-blox receiver and the result showed that the horizontal error RMS was improved by about $24 \%$ from $1.05 \mathrm{~m}$ to $0.8 \mathrm{~m}$ and the vertical error RMS was improved by about $16 \%$ from $1.17 \mathrm{~m}$ to $0.98 \mathrm{~m}$. In particular, the error was decreased by about $1.5 \mathrm{~m}$ in some intervals. Therefore, the DGPS-CP algorithm to which the SBAS correction was applied may solve the problem of incomplete error removal which may take place in the case where the correction of some visible satellites of a user is not provided by a reference station.

\section{REFERENCES}

Jeong, M. 2009, Analysis of MSAS Correction Information and Performance in Korea, Journal of The Korea Society for Aeronautical and Space Sciences, 37, 372-382.

Kaplan, E. D. 2006, Understanding GPS: Principles and Application, 2nd ed. (Boston: Artech House Publishers)

NMEA (National Marine Electronics Association), [Internet], cited 2011 Oct 30, available from: http://www.nmea. org/content/nmea_standards/nmea_0183_v_410.asp

Park, B., Lee, J., Kim, Y., Yun, H., \& Kee, C. 2013, DGPS Enhancement to GPS NMEA Output Data: DGPS by Correction Projection to Position-Domain, The Journal of navigation, 61, 249-264.

Park, B., Lee, J., Kim, Y., Yun, H., \& Song, J. 2011, Algorithm Development of Position-domain DGPS by Correction Projection and its Performance, the 18th GNSS Workshop

Rizos, C. 1999, How Good is GPS? SNAP-UNSW, [Internet], cited 2011 Oct 30, available from: http://www.gmat. unsw.edu.au/snap/gps/gps_survey/chap2/244dgps. htm

RTCA, Inc., 2001, Minimum Operational Performance Standards for Global Positioning System/Wide Area Augmentation System Airborne Equipment, RTCA DO229, Nov 2001.

Sakai, T., Matsunaga, K, \& Hoshinoo, K. 2010, Computing SBAS Protection Levels with Consideration of All Active Messages, Proceedings of the 23rd ION GNSS 2010, 2042-2050.

Shimamura, A. 1999, MSAS (MTSAT Satellite-based Augmentation System) Project Status, Air \& Space Europe, 1, 64-67.

Tsujii, T., Tomita, H., Fujiwara, T., \& Harigae, M. 2008, Preliminary Experiments of GPS/INS Based Integrity Monitoring Using MSAS Differential Correction Data, 17th World Congress Proceeding, IFAC.

Yoon, D. H., Park, B. W., Park, H. W., \& Seo, S. W. 2013, Position area mapping algorithm of correction data using NMEA information of navigation satellite and Comparison of DGPS performance, KGS Conference 2013 Proceeding.

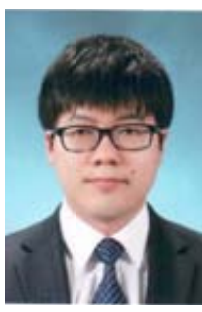

Dong Hwan Yoon is an undergraduate student of Aerospace Engineering in Sejong University. His research interest includes DGPS-CP, GPS correction message protocol, and GPS receiver.

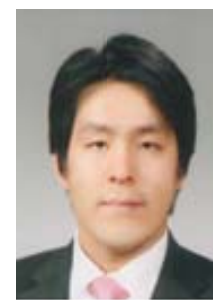

Byungwoon Park received the Bachelor degree, Master degree and Ph.D degree in Mechanical and Aerospace Engineering from Seoul National University, Korea, in 2001, 2003, and 2008. He is currently an assistant professor of Aerospace Engineering in Sejong University. His research interest includes DGPS, WADGPS, RTK, Network RTK, and applications of GPS

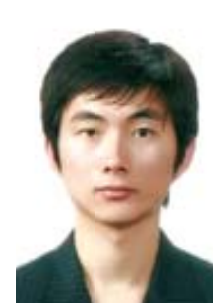

Ho Yun received the Bachelor degree and Ph.D degree in Mechanical and Aerospace Engineering from Seoul National University, Korea, in 2006, 2013. His research interests include WADGPS and integrity monitoring.

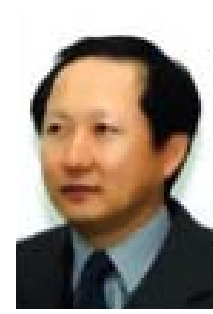

Changdon Kee received the Bachelor degree and Master degree in Mechanical and Aerospace Engineering from Seoul National University, Korea, in 1984, 1986. He received the Ph.d degree in Aeronautics and Astronautics from Stanford University, USA, in 1994. He is currently a professor in Seoul National Universiy. His research interest includes WADGPS, CDGPS, automatic control of Unmaned vehicle, Attitude determination using GPS 